\section{Enteral Nutrition During Noninvasive Ventilation: We Should Go Deeper in the Investigation}

\section{To the Editor:}

We read with interest the paper by Kogo et al. ${ }^{1}$ In our opinion, the delivery of enteral nutrition during noninvasive ventilation (NIV) represents one of the main topics related to this type of respiratory support, and the authors have well addressed this issue in their retrospective study. However, the results of their research raise some concerns and considerations.

The size (diameter) of nasogastric tubes used for enteral nutrition in the NIV group of subjects was not reported. For the reader, this is important information, since smaller diameter nasogastric tubes, even if better tolerated and less prone to esophageal reflux in comparison with the larger ones, ${ }^{2}$ can be easily dislodged, increasing the risk of vomit and inhalation.

Moreover, we have no information about the presence or absence of nasogastric tubes in the no-enteral nutrition NIV group of subjects. Their presence could have affected the comparison of airway complications between the 2 groups and, moreover, the subjects' outcome related to the duration of NIV. In fact, we should take into account that, despite low percentages of reported aspiration pneumonia $(<5 \%)$, aerophagia is a common NIV complication, and gastric insufflation varies from 10 to $50 \% .^{3}$ On the other hand, the insertion of a nasogastric tube can theoretically reduce these adverse effects.

Although the authors described some key points for enteral nutrition administration management during the study, there was a lack of clear standardization regarding the enteral nutrition protocol. ${ }^{1}$ This element, plus the 2 different clinical settings in which the study was performed, could have contributed to some variability in the subjects' outcomes. As an example, there is no mention of the eventual utilization of prokinetic agents and, if so, their administration's timing.

Recent enteral nutrition guidelines by the American Society for Parenteral and Enteral Nutrition (ASPEN) no longer recommend the routine assessment of gastric residual volume on a regular basis for critically ill patients. ${ }^{4}$ In fact, the suggested level of tolerance is now $500 \mathrm{~mL} .{ }^{4}$ However, we believe in a more prudent approach in NIV patients. According to the previous 2009 ASPEN guidelines, gastric residual volume assessment during enteral nutrition should be performed every $4 \mathrm{~h}$ in critically ill patients, and the rate of $250 \mathrm{~mL}$ was the tolerability threshold. ${ }^{5}$ We believe that the authors' approach of an 8-h interval for gastric residual volume checking could have potentially affected the rate of airway complications.

The opioid drug administration described by the authors is not well specified. We do not know which pharmacologic agents and dosages were administered. We cannot identify whether boluses of intravenous morphine were administered, which are usually employed in the treatment of NIV subjects with severe dyspnea and agitation. Given that morphine can produce well-known emetic adverse effects, ${ }^{6}$ data on the episodes of vomiting in the enteral nutrition-NIV group could have been affected by the use (or not) of this medication.

The authors affirmed that the studied sample were treated with CPAP or bi-level NIV mode (spontaneous/timed). ${ }^{1}$ However, in the results section, the number of subjects received CPAP mode and spontaneous/timed mode was not clearly reported. We think that a subgroup analysis of the complications related to different types of NIV settings should have offered important information about the airway complication exposure risk in the presence of a constant airway pressure and during the bi-level ventilation. In our opinion, CPAP appears to be less risky for gastric distention, vomit, and inhalation than any other bi-level mode.

Moreover, according to the results of Kogo et al, ${ }^{1}$ we cannot obtain the peak values of inspiratory positive airway pressure support delivered in both groups of subjects, since they did not report the fourth quartile. This information could have been useful to understand whether, in the last quarter of the delivered pressure values of inspiratory positive airway pressure, the threshold to open the lower esophageal sphincter was overcome. This threshold value is about $20-25 \mathrm{~cm} \mathrm{H}_{2} \mathrm{O} .{ }^{7}$ The consequences of the ventilation with a similar or higher range of pressure are the risks of air ingestion, gastric distention, and vomiting. In effect, readers should not underestimate the risk related to aerophagia, given that it is significantly associated with ventilatorassociated pneumonia in intubated patients (odds ratio $=2.88, P<.01){ }^{8}$
In light of the above mentioned considerations, our opinion is that several variables are present and should be considered, beyond those reported in this study, before concluding that enteral nutrition is a real risk factor for worse outcomes during NIV, when compared with subjects receiving other kinds of artificial nutrition. This concern is supported by the results of subgroup analysis showing statistically different outcome rates only in the group of enteral nutrition NIV and no-enteral nutrition NIV (no airway complications recorded). ${ }^{1}$

In conclusion, we hope that enteral nutrition will be investigated also in patients treated with helmet CPAP. We believe that helmet CPAP could be associated with fewer vomit events and less gastric distention if compared with the mask, especially during pressure support ventilation. In fact, the pressurization in the helmet is slower than in the mask $^{9}$ and the gas flow is not directed straight toward the patient's nose and mouth. Furthermore, another interesting element to be studied is the relationship between the nurse/patient ratio and the rate of airway complications in patients undergoing NIV and enteral nutrition administration.

Stefano Bambi RN MSN Enrica Mati RN

University of Florence

Emergency and Trauma Intensive Care Unit

Careggi University Hospital Florence, Italy

Christian De Felippis RN IMCU Outpatients Saint James Hospital Sliema, Malta

\section{Alberto Lucchini RN \\ General Intensive Care Unit ASST Monza-Ospedale S. Gerardo Hospital University of Milan-Bicocca Milan, Italy}

The authors have disclosed no conflicts of interest.

DOI: $10.4187 /$ respcare.05509

\section{REFERENCES}

1. Kogo M, Nagata K, Morimoto T, Ito J, Sato $\mathrm{Y}$, Teraoka S, et al. Enteral nutrition is a risk factor for airway complications in subjects undergoing noninvasive ventilation for acute 
respiratory failure. Respir Care 2017;62(4): 459-467.

2. Pearce CB, Duncan HD. Enteral feeding. Nasogastric, nasojejunal, percutaneous endoscopic gastrostomy, or jejunostomy: its indications and limitations. Postgrad Med J 2002;78(918):198-204.

3. Carron M, Freo U, BaHammam AS, Dellweg D, Guarracino F, Cosentini R, et al. Complications of non-invasive ventilation techniques: a comprehensive qualitative review of randomized trials. Br J Anaesth 2013; 110(6):896-914.

4. McClave SA, Taylor BE, Martindale RG, Warren MM, Johnson DR, Braunschweig C, et al. Guidelines for the provision and assessment of nutrition support therapy in the adult critically ill patient: Society of Critical Care Medicine (SCCM) and American Society for Parenteral and Enteral Nutrition (A.S.P.E.N.). JPEN J Parenter Enteral Nutr 2016;40(2):159-211.

5. Bankhead R, Boullata J, Brantley S, Corkins M, Guenter P, Krenitsky J, et al. Enteral nutrition practice recommendations JPEN J Parenter Enteral Nutr 2009;33(2):122-167.

6. Breitfeld C, Peters J, Vockel T, Lorenz C, Eikermann M. Emetic effects of morphine and piritramide. Br J Anaesth 2003;91(2): 218-223.

7. Luria O, Reshef L, Barnea O. Analysis of non-invasive ventilation effects on gastric inflation using a non-linear mathematical model. Resuscitation 2006;71(3):358-364.

8. Destrebecq AL, Elia G, Terzoni S, Angelastri G, Brenna G, Ricci C, et al. Aerophagia increases the risk of ventilator-associated pneumonia in critically-ill patients. Minerva Anestesiol 2014;80(4):410-418.

9. Mojoli F, Iotti GA, Currò I, Pozzi M, Via G, Venti A, Braschi A. An optimized set-up for helmet noninvasive ventilation improves pressure support delivery and patient-ventilator interaction. Intensive Care Med 2013; 39(1):38-44.

\section{Enteral Nutrition During Noninvasive Ventilation: We Should Go Deeper in the Investigation-Reply}

\section{In reply:}

We thank Stefano Bambi and colleagues for highlighting these common concerns regarding the introduction of enteral nutrition during noninvasive ventilation (NIV) for acute respiratory failure. In our protocol, we use a nasogastric tube 8-12 French in diameter. Nasogastric tubes were inserted only in the enteral nutrition group. According to the guidelines, ${ }^{1}$ we use prokinetic agents for patients at high risk for aspiration, including those who have had an episode of vomiting or a high gastric volume, although we could not confirm the details of this point retrospectively.

As Bambi et $\mathrm{al}^{2}$ have mentioned, the recent enteral nutrition guideline by the American Society for Parenteral and Enteral Nutrition no longer recommends using gastric residual volume as a routine care criterion for withholding enteral nutrition inappropriately. However, there is a trial suggesting an association between vomiting and a gastric residual volume of $>250 \mathrm{~mL} .{ }^{3} \mathrm{We}$ also need to emphasize that the enteral nutrition protocol we use for ventilated subjects was mainly intended for those receiving invasive ventilation. Vomiting is much more critical for patients with NIV than for those with invasive ventilation, so we routinely assessed gastric residual volume and selected $250 \mathrm{~mL}$ as the tolerability threshold.

We use morphine or fentanyl to relieve patient dyspnea. In our protocol, we started morphine at $0.02 \mathrm{mg} / \mathrm{kg} / \mathrm{h}$ and fentanyl at $0.05-0.1 \mu \mathrm{g} / \mathrm{kg} / \mathrm{h}$ by continuous infusion and increased or decreased the rate depending on the subject's symptoms. ${ }^{4}$ To treat acute changes in dyspnea, we also use bolus infusion at 1-h doses intermittently as tolerated. Although an emetic adverse effect is associated with opioids, one of the 2 subjects who vomited during enteral nutrition was not being administered opioids. Our study also showed no relationship between airway complications and opioid use in univariate analysis. For these reasons, we concluded that enteral nutrition was an independent risk factor of airway complications, although the number of vomiting events was too small to confirm the actual effect of opioids.

In our study, 67 of 107 subjects (63\%) used bi-level NIV mode (spontaneous/timed) and the rate of spontaneous/timed mode to CPAP mode was not significantly different between the enteral nutrition and no-enteral nutrition group (63\% vs $61 \%, P=.86$ ). There was no difference in the rate of airway complications between spontaneous/ timed mode and CPAP mode (49\% vs $35 \%$, $P=.15)$.

As we showed in Table 1, the median (interquartile range) of inspiratory positive airway pressure was $10(8-14) \mathrm{cm} \mathrm{H}_{2} \mathrm{O}$ in the no-enteral nutrition group, and 10 (812) $\mathrm{cm} \mathrm{H}_{2} \mathrm{O}$ in the enteral nutrition group $(P=.93) .{ }^{5}$ Bambi and colleagues suggested the benefit of a helmet interface to reduce airway complications of NIV. The helmet pressurizes gas flow indirectly to the airway and allows patients to expectorate. It could improve clearance of sputum and reduce the risk of aspiration when patients vomit. ${ }^{6}$ In fact, recent evidence suggested a lower intubation rate with a helmet than with a face mask among subjects with ARDS, where half of the included subjects had pneumonia or aspiration. ${ }^{7}$ However, there have been only a limited number of trials, and large, randomized, controlled trials are still needed to provide more robust evidence.

In conclusion, we are confident about our scientific data on enteral nutrition and airway complications in subjects with NIV and our emphasis on the need for special concern. There is no validated strategy, including the helmet or nursing care suggested by Stefano Bambi, to reduce the critical complications of enteral nutrition among patients with NIV. We need more evidence about this topic.

Mariko Kogo MD Kazuma Nagata MD

Department of Respiratory Medicine Kobe City Medical Center General Hospital Kobe, Hyogo, Japan

Takeshi Morimoto MD PhD Clinical Research Center and Department of Clinical Epidemiology Hyogo College of Medicine Nishinomiya, Hyogo, Japan

Jiro Ito MD
Yuki Sato MD
Shunsuke Teraoka MD
Daichi Fujimoto MD
Atsushi Nakagawa MD
Kojiro Otsuka MD
Keisuke Tomii MD
Department of Respiratory Medicine
Kobe City Medical Center General
Hospital
Kobe, Hyogo, Japan

The authors have disclosed no conflicts of interest.

DOI: $10.4187 /$ respcare.05684

\section{REFERENCES}

1. Martindale RG, McClave SA, Vanek VW, McCarthy M, Roberts P, Taylor B, et al. Guidelines for the provision and assessment of nutrition support therapy in the adult critically ill patient: Society of Critical Care Medicine and American Society for Paren- 